\title{
TU/e EmonOWEN

\section{Optimal illumination for local contrast enhancement based on the human visual system}

\section{Citation for published version (APA):}

Wang, H., Cuijpers, R. H., Luo, M. R., Heynderickx, I. E. J., \& Zheng, Z. (2015). Optimal illumination for local contrast enhancement based on the human visual system. Journal of Biomedical Optics, 20(1), [015005]. https://doi.org/10.1117/1.JBO.20.1.015005

DOI:

10.1117/1.JBO.20.1.015005

Document status and date:

Published: 01/01/2015

\section{Document Version:}

Publisher's PDF, also known as Version of Record (includes final page, issue and volume numbers)

\section{Please check the document version of this publication:}

- A submitted manuscript is the version of the article upon submission and before peer-review. There can be important differences between the submitted version and the official published version of record. People interested in the research are advised to contact the author for the final version of the publication, or visit the $\mathrm{DOI}$ to the publisher's website.

- The final author version and the galley proof are versions of the publication after peer review.

- The final published version features the final layout of the paper including the volume, issue and page numbers.

Link to publication

\section{General rights}

Copyright and moral rights for the publications made accessible in the public portal are retained by the authors and/or other copyright owners and it is a condition of accessing publications that users recognise and abide by the legal requirements associated with these rights.

- Users may download and print one copy of any publication from the public portal for the purpose of private study or research.

- You may not further distribute the material or use it for any profit-making activity or commercial gain

- You may freely distribute the URL identifying the publication in the public portal.

If the publication is distributed under the terms of Article 25fa of the Dutch Copyright Act, indicated by the "Taverne" license above, please follow below link for the End User Agreement:

www.tue.nl/taverne

Take down policy

If you believe that this document breaches copyright please contact us at:

openaccess@tue.nl

providing details and we will investigate your claim. 


\section{Biomedical Optics}

\section{Optimal illumination for local contrast enhancement based on the human visual system}

Huihui Wang

Raymond H. Cuijpers

Ming Ronnier Luo

Ingrid Heynderickx

Zhenrong Zheng 


\title{
Optimal illumination for local contrast enhancement based on the human visual system
}

\author{
Huihui Wang, ${ }^{\mathrm{a}, \mathrm{b}}$ Raymond H. Cuijpers, ${ }^{\mathrm{b}}$ Ming Ronnier Luo, ${ }^{\mathrm{a}, \mathrm{c}}$ Ingrid Heynderickx, ${ }^{\mathrm{b}}$ and Zhenrong Zheng, ${ }^{\mathrm{a}, *}$ \\ aZhejiang University, State Key Laboratory of Modern Optical Instrumentation, Department of Optical Engineering, 38 Zheda Road, Hang Zhou \\ 310027, China \\ ${ }^{b}$ Eindhoven University of Technology, Human Technology Interaction Group, School of Industrial Engineering and Innovation Sciences, Den \\ Dolech 2, Eindhoven $5600 \mathrm{MB}$, the Netherlands \\ 'University of Leeds, School of Design, Woodhouse Lane, Leeds LS2 9JT, United Kingdom
}

\begin{abstract}
The observed color of an object is influenced by the spectral distribution of an illuminant impinging upon it. Here we explored a method to obtain optimal illumination spectra for local contrast enhancement based on human vision. First, multispectral imaging was used to measure the spectral reflectance of the sample and color segmentation was used to extract its color features. Then we obtained the target-specific optimal illumination by maximizing the color differences of mutual colors in our sample tissue. To verify the effectiveness of this method, simulated images under the optimized illumination were compared to illumination with the standard illuminant D65 and a cool white light-emitting diode $(5500 \mathrm{~K})$. Results showed that the sample under the optimized illumination had a better perceptual color contrast. ๑ 2015 Society of Photo-Optical Instrumentation Engineers (SPIE) [DOI: 10.1117/1.JBO.20.1.015005]
\end{abstract}

Keywords: surgical illumination; color contrast; optimization.

Paper 140688R received Oct. 21, 2014; accepted for publication Dec. 31, 2014; published online Jan. 27, 2015.

\section{Introduction}

In many applications, light has long been generated with traditional light sources, such as incandescent, fluorescent, halogen, or gas-discharged lamps. These light sources each had their own spectral power distribution (SPD), and changing the spectrum on the fly was impossible. Hence, the spectrum of the light source was as is or, in the best case, optimized for the averaged application in a given application context. A measure most commonly used for expressing the quality of the spectrum of an illuminant is the color rendering index (CRI), ${ }^{1}$ which is calculated from the differences in the chromaticities of eight CIE standard color samples (CIE 1995) when illuminated by a light source and by a reference illuminant of the same correlated color temperature (CCT). CIE A and D series illuminants are used as reference sources. A zero color difference means the test source has very high CRI value. A lower CRI score indicates that some colors may appear unnatural when illuminated by the lamp. As such, it is an important aspect to be considered for lighting conditions relying on high CRI, such as surgical lighting. ${ }^{2}$ However, some applications also benefit from alternative ways of optimizing the SPD of a light source: for example, in retail, the spectrum of the illumination may be adjusted to improve the visual freshness of food or the appeal of other goods. ${ }^{3,4}$

Light-emitting diode (LED) illumination has opened a completely new playground in spectrum optimization. LEDs have emission spectra with narrow peaks at different wavelengths and illuminants with varying spectral compositions become feasible by combining them into a luminaire. By driving multiple LEDs in a luminaire to a different extent, the spectral distribution of the light can be adjusted on the fly. The latter may have advantages in several application contexts. Colored light may be used to generate different atmospheres in homes and offices, ${ }^{5-7}$ and to facilitate healing in hospitals. ${ }^{8}$ In addition, colored light may be used to improve texture visibility, for example, in retail ${ }^{9}$ and in surgical lighting. . $^{10,11}$

Recently, LEDs have been incorporated into high-end lighting for open-wound surgery. ${ }^{12,13}$ In this application area, illuminants are conventionally white with control of both luminance and CCT. Having white light with a high CRI is essential for surgical lighting, as surgeons should be able to reliably assess the color of healthy versus sick tissue. The existing standard (IEC-60601) for surgical lighting, therefore, prescribes that the CRI value of the illuminants (the so-called Ra index) should be $>85 .^{2}$ However, the CRI was developed based on the conventional sources, such as fluorescent lamps and halogen incandescent lamps. It may need revision for the new LED-based light sources, because they have dissimilar spectra from traditional light sources. ${ }^{14,15}$ The CRI score would not correlate with a visual evaluation of the color rendering performance of LED-based white light sources. In addition, they do not support new opportunities with LED-based lighting, namely the improvement of texture visibility by switching the light source between different spectra. Surgeons do not only need to distinguish healthy from sick tissue, but also need to understand where exactly to cut during the operation, so light enabling high local contrast is highly desirable. Spectrum optimization of the light may help to improve such contrast visibility.

Previous research already demonstrated light-based color contrast enhancement for clinical applications. Gono et al. used a xenon lamp and a set of color filters to quantitatively enhance contrast in vascular patterns of tongue, esophagus, and colon tissues. ${ }^{16}$ Wieringa et al. proposed a method to enhance superficial blood vessels below pigmented skin by combining images in different spectral bands. ${ }^{17}$ In both cases, 
the light was optimized beforehand for a particular tissue, while surgical lighting should ideally be able to improve visibility for a wide range of biological tissues. Clancy et al. demonstrated a light-pipe LED light source for endoscopic illumination, which combined the light from four LEDs with different colors. With this light source, these researchers also demonstrated the ability to improve the color contrast of vessels with their surrounding tissue. They also recognized that by carefully adjusting the intensity of each of these four LEDs, the visibility of the tissue structure may be improved over a wide range of tissue colors. However, how to exactly quantitatively derive the optimal linear combination of the intensities of the four LEDs was not discussed.

Several researchers enhanced color contrast by shaping the spectral distribution of the illuminant after having analyzed the reflectance differences between two regions of interest. ${ }^{18,19}$ Building further on this idea, Lee et al. ${ }^{20}$ proposed an algorithm to find the optimal spectral distribution of the illuminant, making color differences in the visual field as large as possible. But the limitation of this method is that it starts from camera-based imaging and, so, actually optimizes the spectral distribution of the illuminant in camera RGB color space. Obviously, differences in a camera RGB color space are not directly related to perceptual color differences. It is well documented in the literature ${ }^{21}$ that differences in RGB color space (e.g., sRGB) do not always correspond well to perceptual color differences. Hence, to optimize the illuminant for visible differences in the color of biological tissue, a perceptually more accurate color space such as CIELAB is needed. Also, real-life tissues contain many color variations and it is not immediately clear which color differences need to be optimized. Lee et al. optimized the spectral distribution of the illuminant for only two different colors in the tissue, whereas, for instance, open-wound surgery would require the distinction of multiple colors present in the tissue. This could be caused by different tissue types, levels of oxygen, and types and severities of symptoms. In the present research, we intend to expand the approach of Lee et al. by calculating the optimal spectral distribution for tissue visibility in a more visually uniform threedimensional color space CIELAB because its three dimensions approximately correlate with the perceived lightness, chroma, and hue of stimuli. ${ }^{22}$ Also, we do not limit ourselves to finding the optimum based on two colors, but calculate the actually required number of colors from a multispectral image. In this study, pig heart was chosen as the sample because of its similarity in physical and chemical compositions to that of a human being. ${ }^{23}$ The light sources used in this study consisted of 11 LEDs that could each be tuned separately to a given intensity. As such, a large flexibility of sources can be created to obtain the optimal illumination. First, a multispectral image of the pig heart was made, on which we used clustering to find the relevant number of different color patches in the tissue. We then applied an algorithm to maximize the mutual differences of these colors to optimize different weights for the intensities of the 11 LEDs.

\section{Illuminant Spectral Distribution Optimization}

Spectral reflectance is the fingerprint of an object. The object's color appearance changes with the illumination in a way determined by the spectral reflectance. Let $l(\lambda)$ represent the SPD of an illuminant, $r(\lambda)$ be the spectral reflectance of a scene point, and $\bar{x}(\lambda), \bar{y}(\lambda), \bar{z}(\lambda)$ be the color matching functions of CIE $X Y Z$ color space. ${ }^{22}$ Then the tristimulus values $X, Y$, and $Z$ are given by

$$
\begin{aligned}
& X=k \sum l(\lambda) r(\lambda) \bar{x}(\lambda) Y=k \sum l(\lambda) r(\lambda) \bar{y}(\lambda) \\
& Z=k \sum l(\lambda) r(\lambda) \bar{z}(\lambda) k=100 / \sum l(\lambda) r(\lambda) \bar{y}(\lambda),
\end{aligned}
$$

where the constant value $k$ is defined such that it results in $Y$ being 100 for the perfect diffuser. Although the CIE $X Y Z$ color space has been widely used, it suffers from a serious disadvantage: the distribution of the colors on it is so nonuniform that it does not correlate with the perception of hue and saturation. To obtain perceptually uniform color coordinates, the tristimulus values were then transformed to $L^{*} a^{*} b^{*}$ values in CIELAB color space. The color difference of two colors $\Delta E_{00}$ was then obtained from the CIEDE2000 equation, ${ }^{24}$ which is demonstrated to predict perceptual color differences more accurately. ${ }^{22}$ If we now suppose that there are $n$ relevant colors in a sample tissue, we can derive the optimal illumination spectrum by maximizing the color contrast, which can be formulated as the following maximization equation:

$\mathbf{L}_{\mathrm{opt}}=\underset{\mathbf{L}}{\arg \max } \sum_{i}^{n} \sum_{j}^{n} \Delta E_{00}(\mathbf{L})_{i j}(i \neq j)$,

where $\Delta E_{00}(\mathbf{L})$ represents the color difference formula CIEDE2000 as a function of the illuminant spectrum vector $\mathbf{L}$. As it is unrealistic to create an arbitrary spectrum, LED light sources can be used to compose the most appropriate illumination. If the spectra of LED sources $\mathbf{I}_{1}, \mathbf{I}_{2}, \ldots, \mathbf{I}_{m}$ are given, the maximization problem of Eq. (2) can be written as follows:

$\mathbf{x}_{\mathrm{opt}}=\arg \max _{\mathbf{x}} \sum_{i}^{n} \sum_{j}^{n} \Delta E_{00}(\mathbf{x I})_{i j}(i \neq j)$,

where $\mathbf{I}$ is a matrix consisting of LED light source vectors $\mathbf{I}_{1}, \mathbf{I}_{2}, \ldots, \mathbf{I}_{m}$ and $\mathbf{x}$ is a vector of weights representing the relative intensities of the LED sources.

In order to obtain an optimal illumination by Eq. (3), three steps are essential: (1) the spectral reflectance data of each point in the scene should be measured, (2) the number $n$ of relevant colors in the tissue should be computed, and (3) the optimized SPD should be calculated. In this study, we used a multispectral imaging system to measure the spectral reflectance of the sample, and we used K-means clustering to determine the number of different relevant colors in the sample.

\subsection{Spectral Reflectance Acquisition}

In multispectral color imaging, the spectral reflectance of a sample can be reliably reconstructed, as shown by several researchers. ${ }^{25,26}$ The system we used has been previously characterized and described. ${ }^{27}$ As illustrated in Fig. 1, the multispectral imaging system consists of a monochrome camera and a filter wheel. The filter wheel contains 11 filters and is installed between the camera and the lens. With this system, the spectral reflectance of a sample can be obtained at pixel-level resolution and with a spectral resolution ranging from 400 to $700 \mathrm{~nm}$ with $10-\mathrm{nm}$ intervals. Figure 2(a) shows the multispectral image of a section 


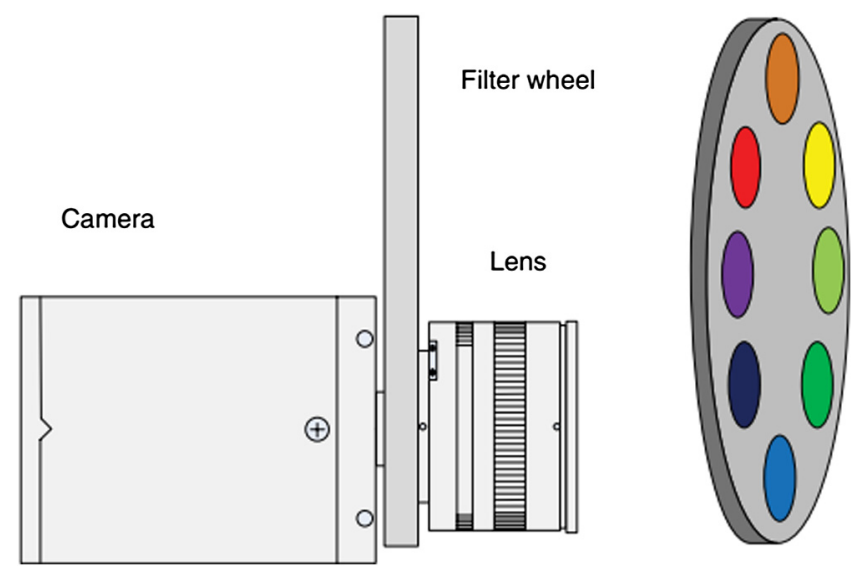

Fig. 1 Schematic illustration of the multispectral imaging system.

of a pig heart with a size of 1040 by 1392 pixels. From each pixel, the spectral reflectance data can be obtained, as shown for three pixels of the image in Fig. 2(b). Hence, color coordinates per pixel can be computed in any color space if the SPD of the illuminant is known.

\subsection{Color Segmentation}

As stated in Sec. 2.1, a multispectral image contains the spectral reflectance data per pixel, so the optimization problem described in Eq. (3) would involve maximizing the sum of mutual color differences within a matrix with 1040 rows and 1392 columns. Since this problem has very high dimensionality, optimization would become very slow. For practical applications, the corresponding accuracy of optimizing color differences between all pixels is unnecessary. Hence, the image was simplified using K-means clustering, where each cluster consists of pixels with similar color coordinates in CIELAB color space.

$\mathrm{K}$-means clustering is one of the most widely used algorithms in many practical applications, like pattern recognition, image processing, and statistics. The technique has been widely discussed and improved by many researchers. ${ }^{28,29}$ Clustering aims at partitioning a given data set into disjoint subsets so that the specific clustering criteria are optimized. The standard clustering criterion of the K-means method is that for each data point, its squared Euclidean distance to the corresponding cluster center is computed and then the sum of these distances over all points in the data set is minimized. In our particular example, we want to obtain colors that can represent the sample, so $\mathrm{K}$-means clustering is used to segment pixels of the multispectral image [shown in Fig. 2(a)] with similar $\mathrm{L}^{*} \mathrm{a}^{*} \mathrm{~b}^{*}$ values into the same cluster. The averaged $\mathrm{L}^{*} \mathrm{a}^{*} \mathrm{~b}^{*}$ values of pixels in the same cluster were used as color coordinates of this cluster. Obviously, if we want to optimize for the human visual system, the computed distances between colors must be perceptually meaningful. That is why CIELAB is used.

To this end, we used the function cvKMeans 2 offered by openCV (version 2.4.4). A drawback of the K-means clustering algorithm is that the cluster number $K$ should be specified. In order to find the optimal cluster number, a set of different $K$ values was explored. Figure 3 shows the clustering results with different $K$ values. The picture labeled Original in Fig. 3 represents the multispectral image shown in Fig. 2(a). As can be seen in Fig. 3, the images resulting from different values of $K$ become closer to the original one as the $K$ value increases. A pilot experiment was conducted by a few observers. They were asked to judge the similarity between the original and the images with different clusters. It was found that the images with 30 clusters consistently gave the lowest cluster number and observers could hardly distinguish the difference. Hence, a $K$ of 30 was assumed to be the appropriate number of clusters needed to represent the multispectral image. Note that this does not necessarily mean that we will also need 30 clusters for the optimization of the illumination, as will be discussed in more detail in Sec. 2.3. Figure 4 shows the spectral reflectance curve of each of the 30 clusters, obtained by averaging the reflectance data of all pixels in the same cluster. For the next step of optimizing the illuminant, clusters existing of overexposed (whitish) pixels and of (gray) background pixels were removed because they would affect the optimization of the illuminant in an uncontrolled way. As such, only 19 clusters were left for further optimization. The reflectance curves of the removed clusters are marked gray in Fig. 4.

\subsection{Illuminant Spectral Distribution Optimization}

The third step in the process consists of optimizing Eq. (3) using the color differences $\Delta E_{00}$ among the 19 remaining clusters. The matrix I is composed of the SPD of 11 LED light sources covering the visible light spectrum from 400 to $700 \mathrm{~nm}$ with a 4-nm interval measured by PhotoResearch PR655 SpectraScan Spectroradiometer, as shown in Fig. 5. Eight of the LEDs are narrow-band colored, whereas three LEDs are white with a different CCT, i.e., 3000, 4000, and 5500 K. Solving Eq. (3) then

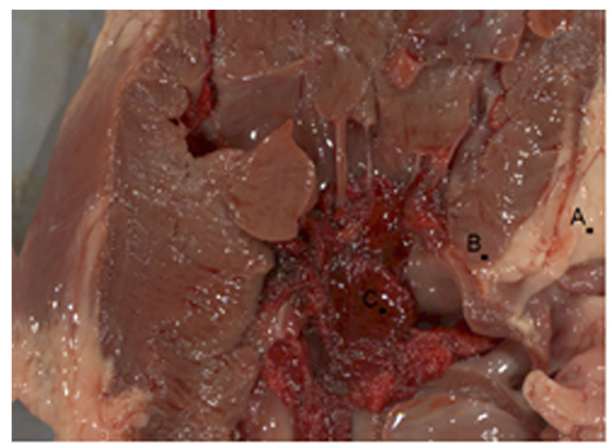

(a)

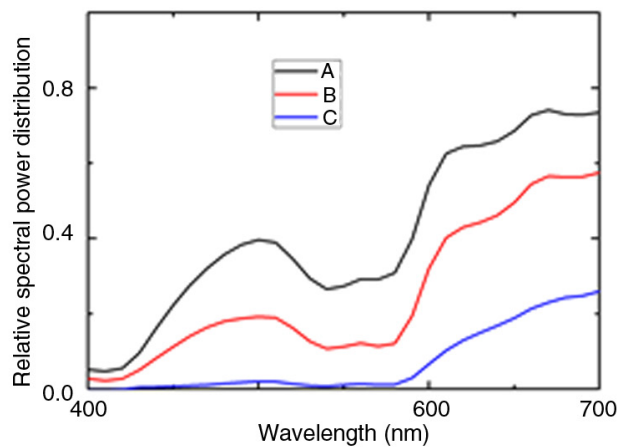

(b)

Fig. 2 (a) Multispectral image of the section of a pig heart (displayed in RGB format). (b) Spectral reflectance curves of pixels $A, B$, and $C$ of the image. 


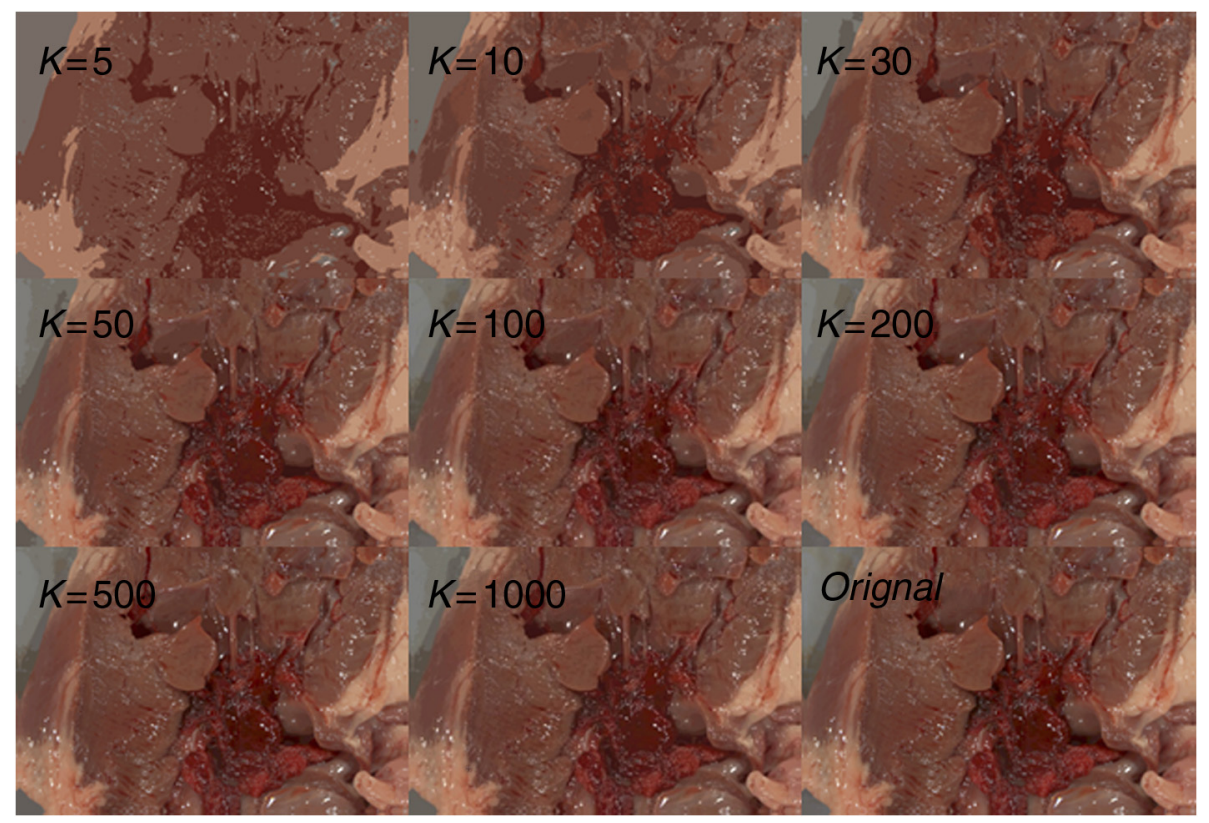

Fig. 3 Results of clustering the pixels of the multispectral image with different values of $K$.

results in the vector $\mathbf{x}$, providing the intensity per LED for which color contrast is maximal.

As Eq. (3) is nonlinear, particle swarm optimization (PSO) was used to find the optimal weight vector $\mathbf{x}$. PSO is a popular search algorithm as it is efficient and has very few parameters to adjust. It can be applied to solve most optimization problems and problems that can be converted to optimization problems. ${ }^{30}$ A disadvantage of the PSO algorithm is that it may find local optimal solutions. In order to obtain a global optimum to Eq. (3), the optimization is run 10 times for each $K$ value, and then the SPD with the maximum color difference over the 10 runs was selected. Figure 6 plots the optimized SPD for each of the 10 different runs when $K=30$. It clearly shows two different optimal solutions that mainly differ in the long-wavelength peak. Apart from that, the optimized SPDs for the different runs are very similar. Results of more than 10 runs were similar with the two local solutions with no improvement of the optimization results.

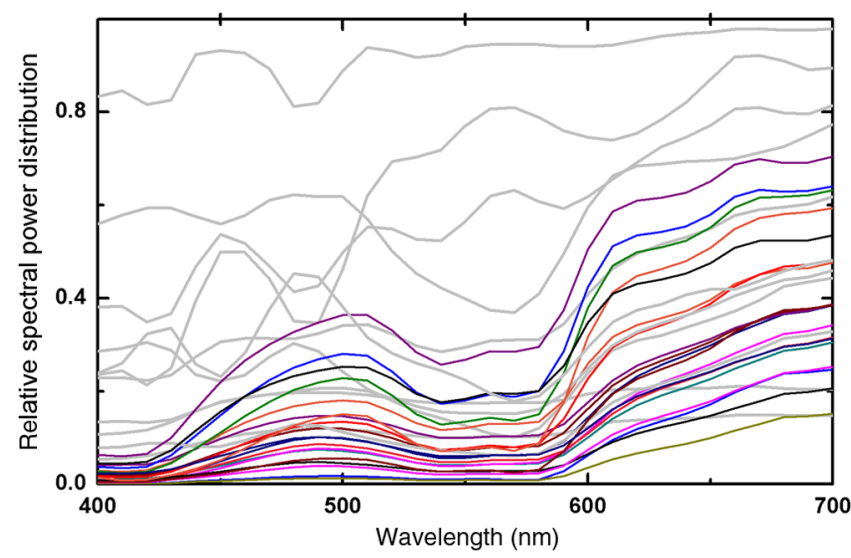

Fig. 4 Spectral reflectance curve of each of the 30 clusters (the curves rendered in gray do not contribute to the color contrast of the pig heart tissue, but belong to overexposed pixels or to the gray background).
Figure 7 shows the SPDs resulting from the optimization of Eq. (3) for different values of $K$. These optimal spectral distributions tend to converge to a single SPD as the $K$ value increases. Only for a $K$ value of 5 is the SPD pattern very dissimilar, as can be seen from the black curve in Fig. 7. For a $K$ value of 10 or higher, the optimal SPD consistently has high luminance in wavelengths around 470, 510, and $650 \mathrm{~nm}$. The latter observation confirms that 30 color clusters are sufficient to represent the color information of the sample tissue. As the difference of optimal SPDs shown in Fig. 7 is so minor that it can affect visual color contrast, the optimization result when $K=30$ was used in the following validation.

\section{Validation of the New Approach}

To validate this new approach of optimizing color contrast, we compare the resulting color contrast of the optimized SPD with that of three alternative illuminants: (1) CIE illuminant D65, (2) a cool white LED with a CCT of $5500 \mathrm{~K}$, and (3) the SPD optimized based on RGB (as proposed by Lee et al. ${ }^{20}$ ) instead of CIELAB. The latter is obtained by maximizing the Euclidean distance in RGB color space-a space that basically is device dependent and, so, not uniquely defined. To solve this issue, we used the sRGB standardized color space. Within this color space, the maximization problem can be formulated as follows:

$$
\begin{aligned}
\mathbf{x}_{\mathrm{opt}}= & \arg \max _{\mathbf{x}} \sum_{i}^{n} \sum_{j}^{n}\left[\left(R_{i}-R_{j}\right)^{2}+\left(G_{i}-G_{j}\right)^{2}\right. \\
& \left.+\left(B_{i}-B_{j}\right)^{2}\right](i \neq j),
\end{aligned}
$$

with $R, G, B$ being the color coordinates of each cluster in SRGB space as a function of the illuminant XI. Figure 8 compares the optimized SPD in sRGB color space (blue curve) to the one optimized in CIELAB color space (black curve). The two SPDs are clearly different; the SPD optimized in the SRGB color space has peak intensities around 440, 520, and $590 \mathrm{~nm}$. Figure 8 also includes the SPD of the two other illuminants used in the comparison, i.e., CIE D65 and LED with CCT of $5500 \mathrm{~K}$. 
Wang et al.: Optimal illumination for local contrast enhancement based on the human visual system

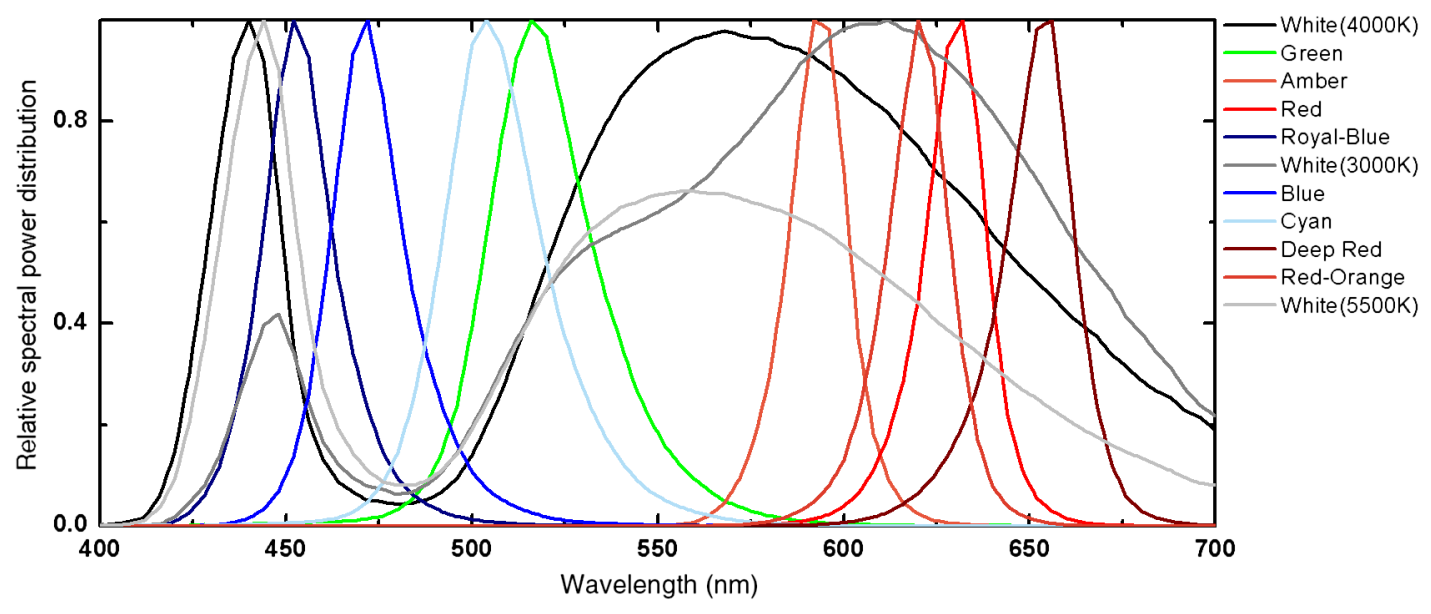

Fig. 5 Relative spectral power distribution of the 11 light-emitting diode (LED) sources used in the current study.

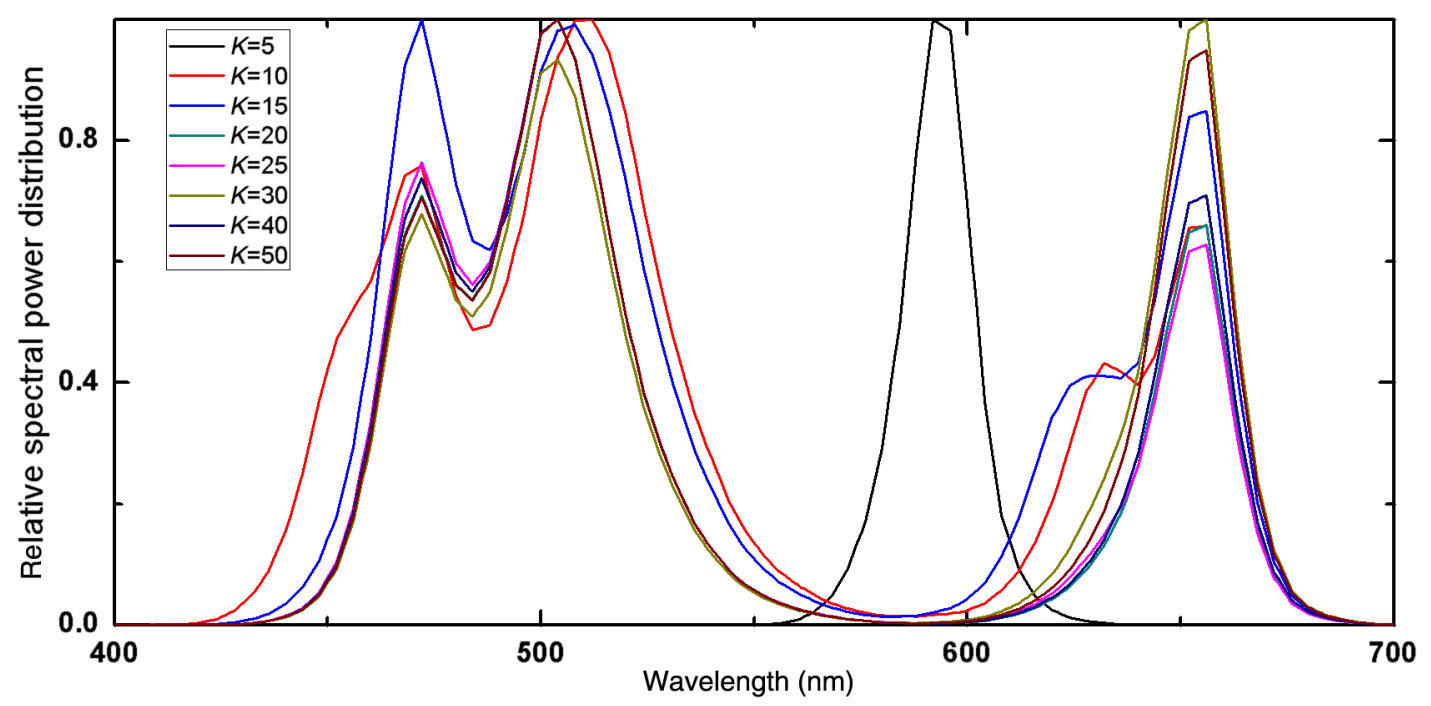

Fig. 6 Spectral power distributions (SPDs) of different runs of particle swarm optimization when $K=30$.

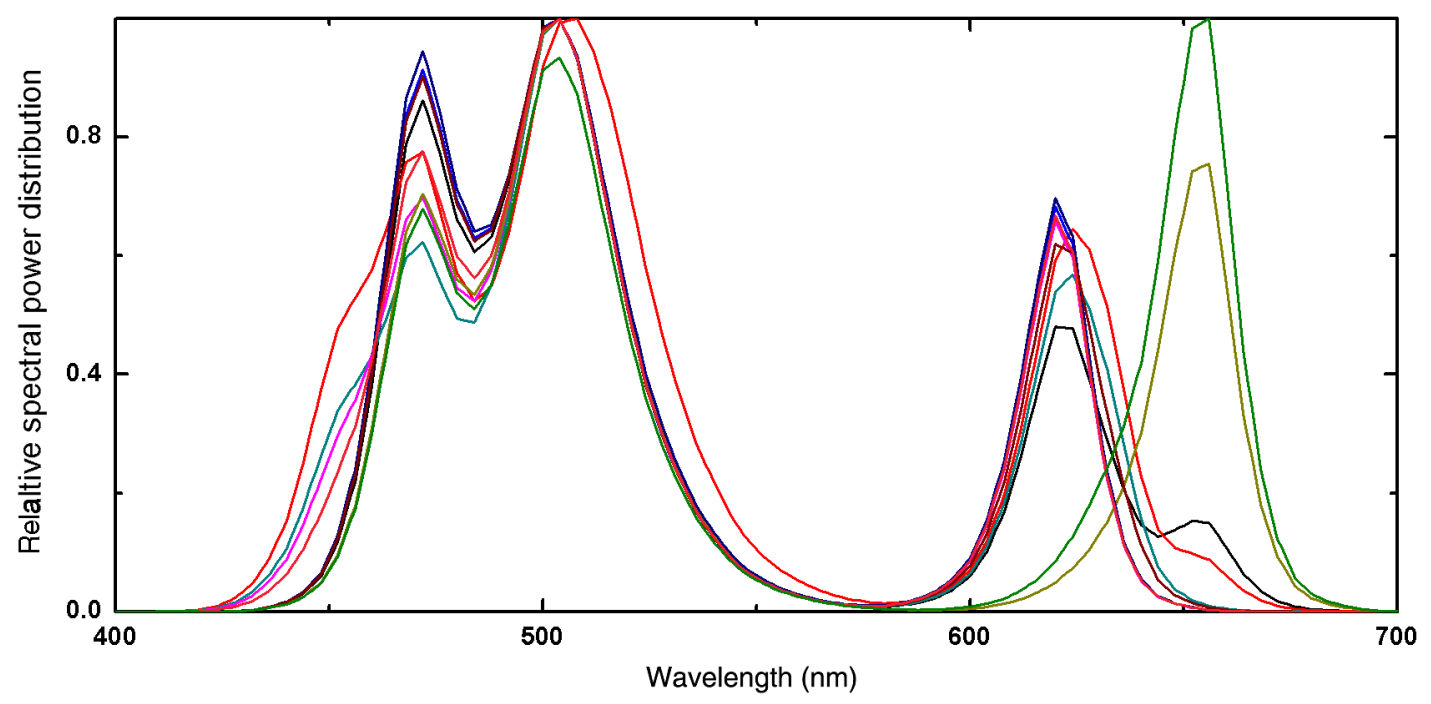

Fig. 7 Optimized spectral distribution of the illuminant for different $K$ values. 


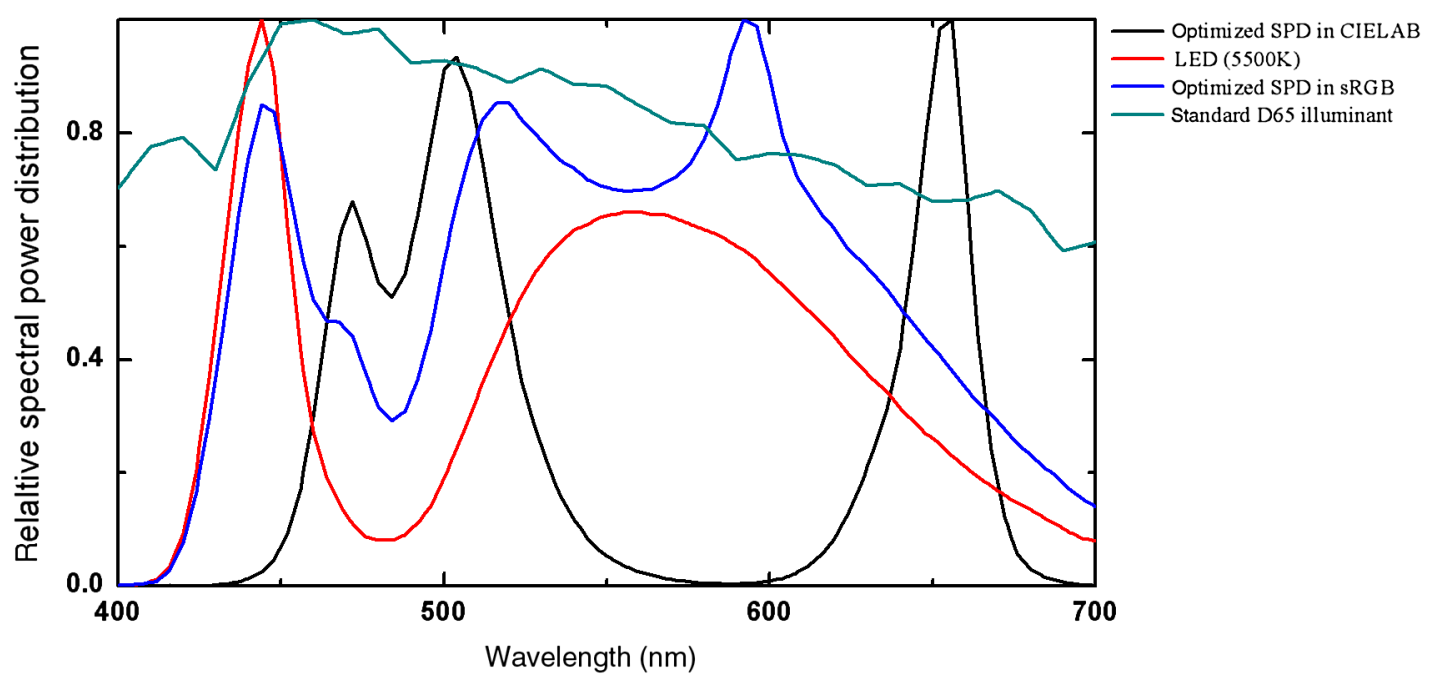

Fig. 8 SPDs of the illuminants used for comparing the color contrast: white LED with correlated color temperature (CCT) of $5500 \mathrm{~K}$ (red curve), D65 (green), optimized illumination in sRGB space (blue), and in CIELAB space (black).

Figure 9 shows the images computed from the multispectral image [of Fig. 2(a)] under different illuminations. The tristimulus values per pixel are obtained from Eq. (1) and then transformed to RGB using the sRGB color space. As shown in Fig. 9, the sample under the illuminant with the SPD optimized in CIELAB color space shows better color contrast than any of the other samples. The color of the sample in itself is bluish under this illuminant and becomes yellowish under the illuminant optimized in SRGB color space or under the LED with a CCT of $5500 \mathrm{~K}$. In the latter two cases, the color contrast is low. The very similar appearance of the sample under the illuminant optimized in SRGB color space and under the white LED with a $\mathrm{CCT}$ of $5500 \mathrm{~K}$ is not surprising, as the two SPDs are also very comparable. To quantify the perceived difference in color contrast, we calculated the sum and mean color differences over all pairs of 19 clusters for the four illuminants under evaluation.

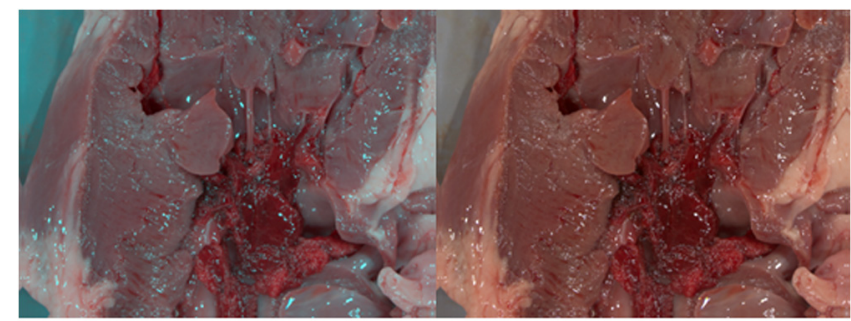

(a)

(b)

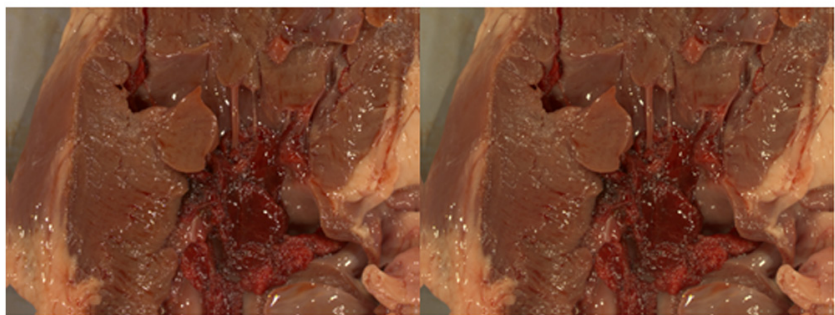

(c)

(d)

Fig. 9 Images computed from the multispectral image assuming four different illuminants: (a) illumination optimized in CIELAB, (b) D65, (c) illumination optimized in SRGB, and (d) white LED with CCT of $5500 \mathrm{~K}$.
These results are summarized in Table 1. It shows a higher mean value for the SPD optimized in CIELAB color space than for any of the other SPDs. Univariate analysis of variance was then used to analyze the difference between mean values under four illuminations after normalization of $\Delta E_{00}$ values. We found a significant effect of illuminant $[\mathrm{F}(3,680)=4.958$, $p=0.002)$. A post hoc analysis with Bonferroni correction revealed that the illumination optimized in CIELAB results in a significantly larger color contrast than the other illuminants $(p<0.027)$. The other illuminants are not significantly different from each other $(p>0.999)$.

\section{Discussion and Conclusions}

This paper proposes an illumination optimization method to effectively enhance the local color contrast of tissue. It is based on a three-step approach of (1) capturing the reflectance data of scene points, (2) clustering these reflectance data in a number of clusters representative of the color information of the scene, and (3) using these clusters to maximize the color differences between them based on the $\Delta E_{00}$ equation in CIELAB color space. We proved the method to be suitable for the optimization of texture visibility in surgical lighting and showed its superiority in terms of color contrast with respect to three alternative illuminants: the illuminant optimized with a similar approach in SRGB color space, a cool white LED, and a CIE D65 illuminant. Particularly, the illuminant optimized in sRGB color space was not really able to improve color contrast within the tissue, giving further support to the knowledge that differences in RGB color space do not necessarily correspond

Table 1 CIEDE2000 values of four illuminations.

\section{White}

light-emitting

$\Delta E_{00}$ diode $(5500 \mathrm{~K})$ D65 Optimized_sRGB Optimized_CIELAB

\begin{tabular}{lllll}
\hline Sum & 2516 & 2564 & 2548 & 3032 \\
Mean & 14.7 & 14.9 & 14.9 & 17.7 \\
\hline
\end{tabular}


well to color discrimination in the human visual system. As such, our novel approach shows two important extensions with respect to the earlier work of Lee et al.: ${ }^{20}$ it allows optimization of color contrast for more than two differently colored regions in the tissue, and it optimizes the color contrast in the perceptually more accurate CIELAB color space.

To come to the proposed method, some choices were made that need further justification or research. We used K-means clustering to find the relevant colors between which the contrast needed to be maximized. A variation in the number of clusters used revealed that 30 clusters were sufficient to visually reproduce the color information in the tissue with sufficient accuracy and that the resulting optimized SPD hardly changed when using more than 10 clusters in the optimization algorithm. To perform the optimization, we first (manually) removed 11 of the 30 clusters that represented the gray background or the whitish highlights in the scene. Obviously, this removal is prone to subjectivity in the algorithm, and further research is needed to automatically select the relevant clusters for optimizing color contrast in whatever tissue.

Optimization of the color contrast is done in this paper based on Eq. (3), which calculates color differences taking into account also luminance differences, but not taking into account the spatial dimensions of the different color clusters. As luminance is an important aspect of contrast perception, we included it in the calculation of $\Delta E_{00}$. Omitting the contribution of luminance differences by only considering differences in $\mathrm{a}^{*}$ and $\mathrm{b}^{*}$ is expected to yield suboptimal results, but obviously needs to be further investigated. Weighting the importance of a color difference between two clusters by the size of the clusters in the overall sum of Eq. (3) may further improve the SPD. This would give more weight to the first clusters detected and gradually reduce the weight of the smaller clusters. To some extent, the optimization algorithm automatically reduced the importance of the smaller clusters, as we saw saturation in the shape of the SPD for more than 10 clusters. On the other hand, adding more weight to the first detected bigger clusters may also have a detrimental effect on the SPD, since it may yield an SPD closer to the one found for $K=5$ in Fig. 6, which clearly is suboptimal. So, the smaller color clusters representing some detailed structure also need to be sufficiently represented in the optimization algorithm.

Our results show that for the sample of a pig heart, the optimal SPD has high luminance in wavelengths around 470, 510, and $650 \mathrm{~nm}$. This SPD can be easily reproduced with a luminaire existing of the combination of the 11 LEDs used in this study. This particular optimized SPD even suggests that fewer LEDs would have been sufficient. Using fewer LEDs, however, may also reduce the flexibility in the final implementation of a surgical lamp. The current optimization is performed on a crosssection of a pig heart, and obviously, more samples of different tissues are needed to explore the required flexibility in SPD to create optimal color contrast for these different tissues. Knowing the changes in optimal SPD for a bigger selection of tissues will reveal the number of LEDs needed in the final implementation of a surgical lamp with high color contrast.

Our approach optimizes color contrast at the expense of the natural appearance of colors in the tissue. As such, it may provide surgeons relevant information on tissue texture, but at the expense of color reliability. Hence, the optimized light source is probably not acceptable for continuous use, but it can be used in combination with an illuminant with very high CRI. Since both
SPDs, in principle, can be provided with a carefully selected combination of LEDs, our method can be used to design an illuminant that may be switched on the fly between optimal color rendering and optimal color contrast for any tissue.

\section{Acknowledgments}

The project is supported by National Nature Science Foundation of China, No. 61327902 and Philips Brain-bridge Project. The authors also would like to thank Professor Huiliang Shen at the Department of Information Science and Electronic Engineering of Zhejiang University for supplying their self-developed multispectral imaging system for the present research.

\section{References}

1. CIE 13.3: 1995, "Method of measuring and specifying color rendering properties of light sources," (1995).

2. IEC, International Standard: Medical Electrical Equipment-Part 2-41 Particular Requirements for the Safety of Surgical Luminaires and Luminaires for Diagnosis, International Electrotechnical Commission, Geneva, Switzerland (2009).

3. Y. Wada, C. Arce-Loperab, and T. Masuda, "Influence of luminance distribution on the appetizingly fresh appearance of cabbage," Appetite 54(2), 363-368 (2010).

4. D. Hinks and R. Shamey, "Review of retail store lighting: implications for color control of products," Color. Technol. 127(2), 121-128 (2011).

5. J. E. Flynn and T. J. Spencer, "The effect of light source color on user impression and satisfaction," J. Illum. Eng. Soc. 6(3), 167-179 (1977).

6. P. R. Boyce and C. Cuttle, "Effect of correlated color temperature on the perception of interiors and color discrimination performance," Light. Res. Technol. 22(1), 19-36 (1990).

7. P. J. M. Custers et al., "Lighting in retail environments: atmosphere perception in the real world," Light. Res. Technol. 42(3), 331-343 (2010).

8. D. Hilary, J. Little, and E. Niemann, "Color and lighting in hospital design," Opt. Laser Technol. 38(4), 343-365 (2006).

9. N. K. Park and C. A. Farr, "Retail store lighting for elderly consumers: an experimental approach," Fam. Consum. Sci. Res. J. 35(4), 316-337 (2007).

10. M. Muto et al., "Improving visualization techniques by narrow band imaging and magnification endoscopy," J. Gastroenterol. Hepatol. 24, 1333-1346 (2009).

11. N. T. Clancy et al., "Development and evaluation of a light-emitting diode endoscopic light source," Proc. SPIE 8214, 82140R (2012).

12. A. J. Knulst et al., "Choosing surgical lighting in the LED era," Surg. Innov. 16(4), 317-323 (2009).

13. A. J. Knulst, L. P. S. Stassen, and C. A. Grimbergen, "Standards and performance indicators for surgical luminaire," Leukos 6(1), 37-49 (2009).

14. Y. Ohno, "Spectral design considerations for color rendering of white LED light sources," Opt. Eng. 44(11), 111302 (2005).

15. CIE 177: 2007, "Color rendering of white LED light sources," (2007).

16. K. Gono et al., "Appearance of enhanced tissue features in narrow-band endoscopic imaging," J. Biomed. Opt. 9(3), 568-577 (2004).

17. F. P. Wieringa et al., "Remote non-invasive stereoscopic imaging of blood vessels: first in-vivo results of a new multispectral contrast enhancement technology," Ann. Biomed. Eng. 34(12), 1870-1878 (2006).

18. Z. Zhu et al., "Effect of color illumination on color contrast in color vision application," Proc. SPIE 7855, 785510 (2010).

19. M. Litorja and B. Ecker, "Use of a spectrally tunable source to explore improvement in chromatic contrast for illumination of tissues," Proc. SPIE 7596, 759607 (2010).

20. M. H. Lee, D. K. Seo, and B. K. Seo, "Optimal illumination for discriminating objects with different spectra," Opt. Lett. 34(17), 26642666 (2009).

21. H. C. Lee, Color Imaging Science, pp. 128-129, Cambridge University Press, Cambridge (2005).

22. M. D. Fairchild, Color Appearance Models, John Wiley \& Sons, Chichester (2013). 
23. V. Tuchin, Tissue Optics: Light Scattering Methods and Instruments for Medical Diagnosis, SPIE Press, Bellingham, Washington (2000).

24. M. R. Luo, G. Cui, and B. Rigg, "The development of the CIE 2000 color difference formula: CIEDE2000," Color Res. Appl. 26(5), 340-350 (2001).

25. Y. Murakami et al., "Spectral reflectance estimation from multi-band image using color chart," Opt. Commun. 188, 47-54 (2001).

26. N. Shimano, "Recovery of spectral reflectance of objects being imaged without prior knowledge," IEEE Trans. Image Process. 15, 1848-1856 (2006).

27. H. L. Shen, H. G. Zhang, and J. H. Xin, "Optimal selection of representative colors for spectral reflectance reconstruction in a multispectral imaging system," Appl. Opt. 47(13), 2494-2502 (2008).

28. Y. Deng, B. S. Manjunath, and H. Shin, "Color image segmentation," in Proc. of IEEE Computer Society Conf. on Computer Vision and Pattern Recognition, Vol. 2, pp. 446-451 (1999).

29. D. T. Pham, S. S. Dimov, and C. D. Nguyen, "Selection of K in K-means clustering," Proc. Instn. Mech. Eng. 219(1), 103-111 (2005).

30. R. C. Eberhart and Y. Shi, "Particle swarm optimization: developments, applications and resources," in Proc. of the 2001 Congress on Evolutionary Computation, Seoul Vol. 1, pp. 81-86, IEEE (2001).
Huihui Wang is a PhD candidate at Zhejiang University and Eindhoven University of Technology. Her current research interests include interaction between light and material, and measurement and application of light-emitting diode light.

Raymond Cuijpers is associate professor at Eindhoven University of Technology. His research interests include visual perception, human robot interaction, and human motor control.

Ming Ronnier Luo is a professor of color and imaging science at Zhejiang University and Leeds University. His research interests are image device and image quality, appearance measurement, applied LED Lighting, and digital archiving and restoration.

Ingrid Heynderickx is a full-time professsor at Eindhoven University of Technology, guest research professor at Southeast University of China and part-time professor at Delft University of Technology. She is involved in various research areas: optimization of the optics of LCDs, functional evaluation of personal care devices, and perceptive evaluation of display and lighting systems.

Zhenrong Zheng is a professor at Zhejiang University and part-time professor at Eindhoven University of Technology. His research interest includes optical system design, freeform imaging, and display technologies. 